\title{
Engine fault feature extraction based on order tracking and VMD in transient conditions
}

\author{
Gang Ren ${ }^{1}$, Jide $\mathrm{Jia}^{2}$, Jian $\mathrm{Mei}^{3}$, Xiangyu Jia ${ }^{4}$, Jiajia Han ${ }^{5}$ \\ 1, 4, 5 Fifth Cadet Brigade, Army Transportation University, Tianjin, China \\ ${ }^{2,3}$ Projection Equipment Support Department, Army Transportation University, Tianjin, China \\ ${ }^{3}$ Corresponding author

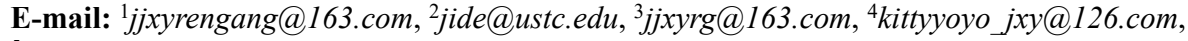 \\ 5474689816@qq.com
}

Received 8 April 2018; accepted 20 April 2018 DOI https://doi.org/10.21595/vp.2018.19902

Check for updates

Copyright $(\mathrm{C} 2018$ Gang Ren, et al. This is an open access article distributed under the Creative Commons Attribution License, which permits unrestricted use, distribution, and reproduction in any medium, provided the original work is properly cited.

\begin{abstract}
A method based on order tracking and Variational Mode Decomposition was proposed to solve the problem of non-stationarity and background noise in the vibration signal of engine. For the vibration signal of diesel engine crankshaft bearing fault and Connecting rod bearing of gasoline engine, the non-stationary signal in the time domain was converted into a pseudo stationary signal on the angular domain based on order tracking. Then the reconstructed signal was decomposed by several components by VMD, and the component that contains fault information was selected. The three-dimensional spectral array of order, speed and power spectrum was calculated, and the fault feature was extracted. The effectiveness of the proposed method is verified by simulation and experiment.
\end{abstract}

Keywords: order tracking, VMD, transient operation, engine, feature extraction.

\section{Introduction}

Most of the engines operate under transient conditions. The diagnosis of engine faults under transient conditions is always a research hotspot. The vibration excitation of engine is mainly caused by the frequency of rotation and the frequency of ignition. Because the running speed of the engine is constantly changing, the fault frequency contained in the vibration signal also changes. It is difficult to extract the fault feature effectively by analyzing the traditional time domain and frequency domain signals. The order tracking technology resamples the vibration signal in an equal angle way by tracking the running speed of the engine. Then the transient signal in the time domain is converted into a stable working condition signal on the angular domain, so that the fault features associated with the speed of the engine are accurately extracted from the engine vibration signal. Reference [1] proposed a novel order tracking method based on discrete spectrum correction technique to analyze wind turbine gearbox vibration for the purposes of health monitoring and fault diagnosis. Reference [2] proposed an online tacholess order tracking technique based on generalized demodulation to conduct the bearing fault detection under speed variation conditions without a tachometer.

However, both the above methods need to be applied under uniform acceleration. In the actual operation of the engine, it is difficult to ensure this. Therefore, the engine acceleration signal is segmented according to the speed and rearranged the acceleration signal. A reconstitution signal similar to uniform acceleration can be obtained by eliminating the repetitive signal. This method is suitable for vibration signal analysis under any condition.

The vibration signal of engine is composed of multi-component complex signals, and its amplitude varies with time. For the complex multi-component signal, it is usually necessary to decompose it into a number of single-component AM-FM signals, and each component is analyzed to extract amplitude and frequency information. Huang et al proposed the Empirical Mode Decomposition (EMD) [3]. EMD has been widely used in mechanical fault diagnosis since it was proposed [4]. EMD is fundamentally divorced from the Fourier transform. EMD analyzes the signal from itself and it is completely adaptive and unsupervised. But there are some problems 
for EMD, such as endpoint effect and modal aliasing.

In recent years, Konstantin Dragomiretskiy [5] proposed a new variable adaptive decomposition method, Variational Mode Decomposition (VMD). The method is essentially composed of a plurality of adaptive Wiener filter and has good noise robustness. Compared with EMD, VMD has strong mathematical theory basis. At the same time, VMD rejects the method of recursive screening stripping. So VMD can effectively alleviate or avoid a series of problems which appear in EMD and has high operation efficiency of decomposition [6]. VMD has been fully utilized in the field of engineering.

After a lot of research, a new method of engine fault diagnosis based on order tracking and VMD is presented in this paper. The proposed method is applied to diesel engine crankshaft bearing fault and gasoline engine connecting rod bearing fault diagnosis under transient conditions. The fault feature is successfully extracted, which verifies the practicability and effectiveness of the method.

\section{Feature extraction based on order tracking and VMD}

\subsection{Order tracking technique}

Assume that the rotation of engine crankshaft is the one with constant acceleration, the rotation angle and time satisfy the following binomial relation:

$\theta(t)=b_{0}+b_{1} t+b_{2} t$

where $\theta(t)$ is the rotation angle of crankshaft; $t$ is time; $b_{0}, b_{1}$ and $b_{2}$ are polynomial coefficients respectively. For vibration signals, the sampling point of each vibration signal segment in a rotation period is related to the rotation angle. As long as each rotation period is sampled at a constant angle, the polynomial coefficients in the Eq. (1) can be solved by fitting the arrival time $\left(t_{1}, t_{2}, t_{3}\right)$ of the three-continuous equal angle sampling points:

$\left[\begin{array}{l}\theta_{1} \\ \theta_{2} \\ \theta_{3}\end{array}\right]=\left[\begin{array}{lll}1 & t_{1} & t_{1}^{2} \\ 1 & t_{2} & t_{2}^{2} \\ 1 & t_{3} & t_{3}^{2}\end{array}\right]\left[\begin{array}{l}b_{1} \\ b_{2} \\ b_{3}\end{array}\right]$,

where $\theta_{1}, \theta_{2}$ and $\theta_{3}$ is the angle interval of the speed signal. Eq. (2) is calculated using Matlab, and the corresponding time at each angle is obtained:

$t_{k}=\frac{1}{2 b_{2}}\left(\sqrt{b_{1}^{2}++4 b_{2}\left(\theta_{k}-b_{0}\right)}-b_{1}\right)$

where $\theta_{k}$ is the corner position; $t_{k}$ is the corresponding time of the corner position. According to the Eq. (3), the time of resampling can be obtained, and the resampling signal can be obtained by three spline interpolation algorithm. The FFT is used to calculate the resampling signals of multiple segments (each segment of the signal corresponding to a certain rotation period), and the three-dimensional spectrum of order, rotational speed and power spectrum can be obtained to achieve the purpose of order tracking.

\subsection{Variational mode decomposition}

The VMD algorithm defines the intrinsic mode function as a non-stationary AM-FM signal. The intrinsic mode is considered as follows: 
$u_{k}(t)=A_{k}(t) \cos \left(\varphi_{k}(t)\right)$

where the phase $\varphi_{k}(t)$ shall satisfy the following condition: $\varphi_{k}(t) \geq 0$; the envelope line $A_{k}(t)$ should satisfy the following condition: $A_{k}(t) \geq 0$; the instantaneous frequency $\omega_{k}(t)$ should satisfy the following condition: $\omega_{k}(t)=\varphi_{k}(t) . A_{k}(t)$ and $\omega_{k}(t)$ change slowly, and $\varphi_{k}(t)$ changes more rapidly.

The Hilbert transform is performed for each modal function $u_{k}(t)$, and exponential correction is applied to obtain $K$ modal functions. Then the frequency spectrum of the modal function is corrected to the estimated central frequency, and the bandwidth of the modal component is calculated by using Gauss smoothing. The variational constraint problem can be defined as follows:

$\min _{\left\{u_{k}\right\},\left\{w_{k}\right\}}\left\{\sum_{k=1}^{K}\left\|a_{t}\left\{\left[\delta(t)+\frac{j}{\pi t}\right] * u_{k}(t)\right\} e^{-j \omega_{k} t}\right\|_{2}^{2}\right\}, \quad$ s.t. $\sum_{k=1}^{K} f(t)=u_{k}(t)$,

where $u_{k}$ is the modal component, $\omega_{k}$ is the central frequency for the modal component, $\delta(t)$ is the unit pulse function, and $*$ is the convolution symbol.

In the VMD algorithm, the secondary penalty factor and the Lagrangian multiplication operator are used. Then, the alternating direction method is introduced. $u_{k}^{n+1}, \omega_{k}^{n+1}$, and $\lambda^{n+1}$ are constantly updated, so that the optimal solution of the variational constraint problem can be solved. The expression for the modal component $u_{k}^{n+1}$ is:

$u_{k}^{n+1}=\underset{u_{k} \in X}{\operatorname{argmin}}\left\{\alpha\left\|a_{t}\left\{\left[\delta(t)+\frac{j}{\pi t}\right] * u_{k}(t)\right\} e^{-j \omega_{k} t}\right\|_{2}^{2}+\left\|f(t)-\sum_{i} u_{i}(t)+\frac{\lambda(t)}{2}\right\|_{2}^{2}\right\}$,

where $\alpha$ is the penalty factor, and $\lambda$ is the Lagrange multiplier.

The expression for the modal component $u_{k}^{n+1}$ in frequency domain is:

$\hat{u}_{k}^{n+1}(\omega)=\left(\hat{f}(\omega)-\sum_{i} \hat{u}_{i}(\omega)+\frac{\hat{\lambda}(\omega)}{2}\right) \frac{1}{1+2 \alpha\left(\omega-\omega_{k}\right)^{2}}$.

where $\omega_{k}$ is the center of the modal component power spectrum. The Wiener filter is introduced, which makes the VMD algorithm have better noise robustness.

Similarly, the expression for the central frequency $\omega_{k}^{n+1}$ is:

$\omega_{k}^{n+1}(\omega)=\frac{\int_{0}^{\infty} \omega\left|\hat{u}_{k}(\omega)\right|^{2} d \omega}{\int_{0}^{\infty}\left|\hat{u}_{k}(\omega)\right|^{2} d \omega}$

The stopping condition of the iteration is:

$\frac{\sum_{k=1}^{K}\left\|\hat{u}_{k}^{n+1}-\hat{u}_{k}^{n}\right\|_{2}^{2}}{\left\|\hat{u}_{k}^{n}\right\|_{2}^{2}}<e$.

The VMD algorithm is a linear transformation, so the signal can be reconstructed. The reconstructed signal can be represented as: 
$\hat{f}(t)=\sum_{k=1}^{K} \hat{u}_{k}$.

where $\hat{u}_{k}$ is the final modal component, after the iteration is stopped.

\subsection{Proposed method}

First, the engine vibration signal is segmented according to the rotational speed and rearranged the vibration signal. A restructure signal similar to uniform acceleration can be obtained by eliminating the repetitive signal. Then, the order tracking technique is used to resample the signals in equal angles and turn the time-varying signals into stationary signals in the angular domain. Finally, the VMD is used to decompose the resampling signal into several modal components. According to the correlation coefficient between modal component and vibration signal, the modal component with larger correlation coefficient is selected as the fault component. The order spectrum is calculated, and the fault information is extracted.

\section{Experimental results}

\subsection{Experiment condition}

The structure of engine is complex, and the working environment is abominable. As a result, it is prone to malfunction. The crankshaft bearing is located inside the engine, so it is difficult to diagnose the fault. In this paper, vibration signals are collected from the vibration sensors on the experimental stand. At the same time, the rotational speed sensor signal is collected to calculate the speed of diesel engine. The engine on the experimental stand is Cummins 6BT diesel engine.

The fourth crankshaft bearings of Cummins EQ6BT diesel engine are set with different clearance $(0.10 \mathrm{~mm}, 0.26 \mathrm{~mm}, 0.40 \mathrm{~mm}, 0.55 \mathrm{~mm})$ to simulate the normal, slight, moderate and heavy wear of the crankshaft bearing. Usually, when the engine is accelerating, its fault characteristics are more obvious. In order to ensure the repeatability and stability of the signal, this paper uses the way of fixed speed triggering to collect signals. According to the experience of the maintenance experts, the trigger speed is set to $1800 \mathrm{r} / \mathrm{min}$. When the rotational speed rises to $1800 \mathrm{r} / \mathrm{min}$, the data acquisition is started. Vibration signals are collected on the left side of the fourth main bearings on the surface of the engine block. The sampling frequency is $20000 \mathrm{~Hz}$ and the sampling points are 50000 points. Testing temperature is important when acquiring vibration signals. In the experiment, the temperature of cooling water is measured to reflect the internal temperature of diesel engine. The temperature is controlled at 60-70 degrees $\mathrm{C}$.

\subsection{Experimental data processing}

For order tracking technology, rotational speed signal is very important. Only the accurate speed signal can be used to analyze the order of transient conditions. In the process of experiment, rotational speed is controlled by human and there are uncontrollable factors. Taking the normal wear state as an example, in Fig. 1(a), the whole acceleration process is not uniformly accelerated, and the fluctuation of the rotational speed is large, which is bound to affect the analysis results. The proposed method sorted the rotational speed and eliminated the segments with the same rotational speed. A modified rotational speed is obtained, which is a relatively uniform acceleration process, as shown in Fig. 1(b).

Similarly, the corresponding vibration signals should be corrected appropriately. Through the solution of Eq. (2), the corresponding time of equal angle resampling is calculated, and the vibration signal of equal angle resampling is obtained by three times spline interpolation. Similarly, the proposed method is applied to deal with the vibration signals of other wear states, and the resampling signals are obtained. 
In order to eliminate the background noise, $\mathrm{VMD}$ is used to decompose the equal angle resampling signals. According to the correlation coefficient between modal component and vibration signal, the modal component with larger correlation coefficient is selected as the fault component. The order spectrum of fault component is calculated respectively, and the order, rotational speed and power spectrum constitute the 3D order spectrum array, as shown in Fig. 2.

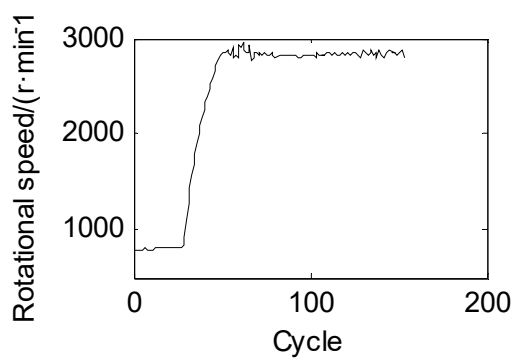

a) Original speed

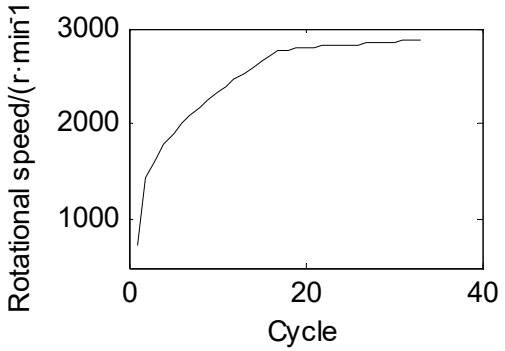

b) Corrected speed

Fig. 1. The curves of rotational speed

From Fig. 2, it can be seen that for different wear states, the impact energy of vibration signals is mainly located in the order $0-20$. When the rotational speed is lower than $2800 \mathrm{r} / \mathrm{min}$, the energy of the order 10 decreases irregularly, with the increase of the wear degree of the crankshaft bearing. When the rotational speed is higher than $2800 \mathrm{r} / \mathrm{min}$, the energy of the order 10 increases in turn, which can be used as a basis for judging the wear fault. Therefore, the proposed method can effectively extract the fault features of diesel engine crankshaft bearings under transient conditions.

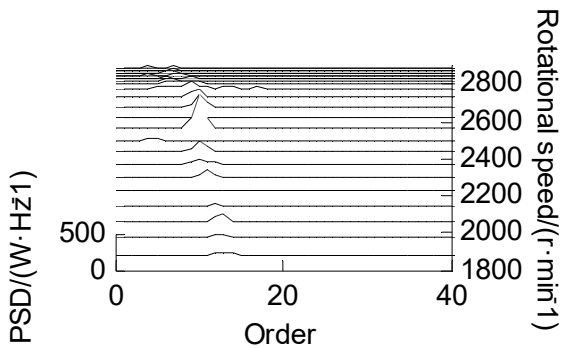

a) Normal wear

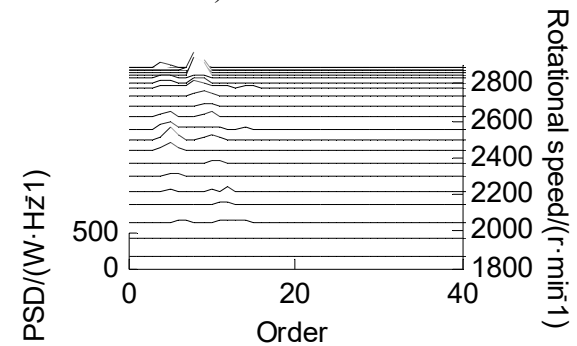

c) Moderate wear

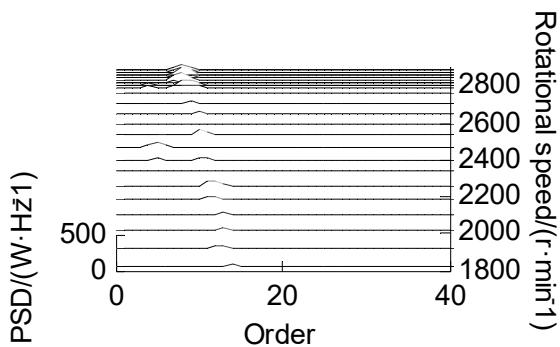

b) Slight wear

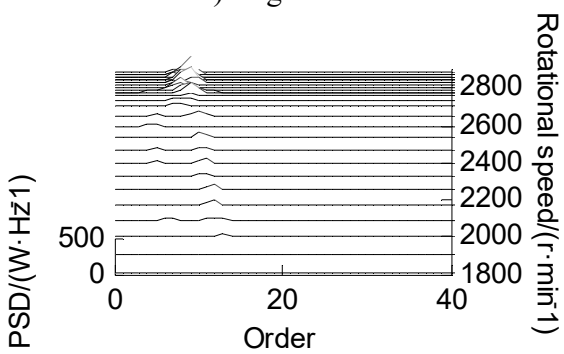

d) Severe wear

Fig. 2. Order tracking chart of different wear state after VMD

\section{Conclusions}

In this paper, a new method based on order tracking and VMD is proposed to analyze the fault of engine transient condition. First, the engine vibration signal is segmented according to the rotational speed and rearranged the vibration signal. A restructure signal similar to uniform acceleration can be obtained by eliminating the repetitive signal, which is applicable to any 
working condition of the engine. Then the non-stationary signal in the time domain is transformed into a pseudo stationary signal on the angular domain, which reduces the influence of speed fluctuation on vibration signal. Finally, the VMD is used to decompose the resampling signal into several modal components. According to the correlation coefficient between modal component and vibration signal, the modal component with larger correlation coefficient is selected as the fault component. The order spectrum is calculated, and the fault information is extracted, and the fault features of the engine are extracted successfully. The experimental verification of the engine shows that the proposed method is effective.

\section{References}

[1] He G., Ding K., Li W., et al. A novel order tracking method for wind turbine planetary gearbox vibration analysis based on discrete spectrum correction technique. Renewable Energy, Vol. 1971, 2016, p. 364-375.

[2] Wang Y., Xu G., Luo A., et al. An online tacholess order tracking technique based on generalized demodulation for rolling bearing fault detection. Journal of Sound and Vibration, Vol. 367, Issue 2016, 2016, p. 233-249.

[3] Huang N. E., Shen Z., Long S. R. The empirical mode decomposition and the Hilbert spectrum for nonlinear and non-stationary time series analysis. Proceedings Mathematical Physical and Engineering Sciences, Vol. 454, Issue 1971, 1998, p. 903-995.

[4] Yu D., Cheng J., Yang Y. Application of EMD method and Hilbert spectrum to the fault diagnosis of roller bearings. Mechanical Systems and Signal Processing, Vol. 19, Issue 2, 2005, p. 259-270.

[5] Dragomiretskiy K., Zosso D. Variational mode decomposition. IEEE Transactions on Signal Processing, Vol. 62, Issue 3, 2013, p. 531-544.

[6] Zhao C., Feng Z. P. Application of multi-domain sparse features for fault identification of planetary gearbox. Measurement, Vol. 104, 2017, p. 169-179. 